\section{Psychological and Physiological Influences of A Head-Down Tilted Condition}

\author{
Miwako Kato ${ }^{1}$, Yasuhiro Itoh ${ }^{2 \star}$, Jun Shimizu ${ }^{3}$, \\ Shinobu $\mathrm{Naga}^{3}$, Akira Takabayashi ${ }^{2}$, \\ and Shunji Nagaoka ${ }^{2}$ \\ ${ }^{1}$ Graduate School of Psychology Studies, Aichi \\ Shukutoku University, Nagakute, Aichi 480-1197, \\ Japan \\ ${ }^{2}$ Fujita Health University Graduate School of Health \\ Sciences, Toyoake, Aichi 470-1192, Japan \\ ${ }^{3}$ Faculty of Psychology Studies, Aichi Shukutoku \\ University, Nagakute, Aichi 480-1197, Japan
}

\section{Abstract}

The aim of this study was to evaluate psychophysiological effects of two postures on emotional states, in relation to the autonomic responses obtained from measures of heart rate variability and salivary cortisol. This study compared a sitting posture with a 7 degree head-down-tilted ( $7^{\circ}$ HDT) condition. The study assessed anxiety states during the HDT conditions. Twenty-eight healthy volunteers (university students) participated (age $=20.9 \pm 1.5$ ). The participants were divided into two groups, high-anxiety and normal, based on state trait anxiety inventory (STAI) scores. A simple protocol for monitoring electrocardiograms (ECGs) was applied for all participants, such that they first quietly adopted the sitting posture for $\mathbf{2 0}$ minutes, followed by a subsequent $7^{\circ}$ HDT posture was then assumed on a tilting bed for another 20 minutes. At the end of each period, a saliva sample was collected from the participant to measure cortisol concentration and salivary buffering action. Affect-grid scoring was applied to evaluate participant's emotional states. Heart rate variability was calculated to evaluate the balance of autonomic nervous system activities (LF/HF). When the HDT posture was assumed, the high anxiety group showed significantly higher salivary cortisol levels, increases in 0.39 $\pm 0.35 \mu \mathrm{g} / \mathrm{dl}$ from $0.24 \pm 0.22 \mu \mathrm{g} / \mathrm{dl}(p<0.05)$, as well as LF/HF ratios when compared to the sitting posture, increases in $32.68 \pm 42.98$ from $6.89 \pm 9.80(p<0.05)$. However, salivary buffering action did not show any significant changes across the postures or groups. Participant's

Received: August 2, 2010, Accepted: October 14, 2010

*To whom correspondence should be addressed:

Tel: +81-(0)562-93-2520; Fax: +81-(0)562-93-4595;

E-mail: yasitoh@fujita-hu.ac.jp emotion, both of pleasantness and arousal, showed significant changes between postures. These results suggest that the STAI is a useful component of a psycho-physiological evaluation gravitational tolerance and/or adaptability to a microgravity environment. (C)2010 Jpn. Soc. Biol. Sci. Space; Article ID: 102402007

Key words: Head-down tilted (HDT), Anxiety, Autonomic nervous response, Salivary cortisol concentration, Affection

\section{Introduction}

Astronauts must experience a microgravity environment when staying in space, and presumably experience several distinct emotional states depending on time and individual characteristics. Although considerable exploratory efforts have been made in the fields of space physiology and psychology, the psychological effects of space flight remain highly variable and cannot be predicted for individual astronauts. It is also reported that emotional states during space flight can change remarkably within a week, or during the so-called early adaptation phase (Mano, 2004; Iwase, 2002; Shimizu, 2000). The first phase of early adaptation may produce many problems for astronauts, which arise from vestibular dysfunction, instability of visuo-motor coordination, and spatial disorientation, a constellation of difficulties known as space adaptation syndrome. Typically, the majority of such difficulties cease within a week. Over the past few decades, a considerable number of efforts have been made to characterize the physiological basis of anxiety (Candland, 1962). In the present study, we define anxiety as the psychological/emotional state that occurs in a novel situation in which some hazardous or unpleasant condition is expected (Kamimura, 2001).

Our study may prove useful in efforts to evaluate and manage this emotional state.

\section{Materials and Methods}

\section{Participants}

The participants tested were 28 healthy university student volunteers (mean age $=20.9 \pm 1.5$ years), including three males. All participants provided their informed consent. The participants were divided into two groups (high-anxiety and normal) based on STAI scores (Spielberger, 1962; Nakazato, 1982). The high anxiety group (>42 points) included 12 females and 1 male, with an average age of $20.7 \pm 0.9$ years. All other participants were in the 'normal group', which included 13 females and 2 males, with an average age of $21.1 \pm 1.8$ years. The STAI is a standardized measure with high levels of reliability and validity. The standard value thought to be associated with high levels of anxiety was set at 42 points.

\section{Procedure}

Electrocardiograms (ECGs) were monitored, first during a quiet sitting posture for 20 minutes, and then 
subsequently during a $7^{\circ}$ HDT posture on a tilting bed for another 20 minutes. Based on the results of some preliminary experiments, autonomic nervous system responses become stable after fifteen minutes subsequent to changing posture. At the end of each period, a saliva sample was collected from each participant to measure cortisol concentrations, and affect-grid scoring was applied to evaluate emotional states (Shimizu et al., 1999; Russell et al., 1989). In the present study, pleasantness and arousal were measured by using the Affect-grid method. The saliva collected was placed in a Salivette container (SARSTEDT, Germany) without citric acid. Saliva collection was planned for between 1 p.m. and 4 p.m. to minimize variation caused by circadian rhythms, with single timed stimulation for 1.5 minutes to total saliva obtained (Kawamura, 1959). The protocol required neither eating nor drinking (except water) for one hour prior to saliva collection, so that food debris would not stimulate salivation. After saliva sampling, the Salivettes were centrifuged as soon as possible for 5 minutes at $\times 2800 \mathrm{G}$. Clear supernatant was used for immediate $\mathrm{pH}$ measurement. For the cortisol measurement, all samples were kept frozen at less than $-20^{\circ} \mathrm{C}$ until assay.

\section{measurement.}

ECG measurement was carried out using a digital polygraph, MP30B-CE (BIOPAC Systems, Goleta USA), with a sampling rate of $500 \mathrm{~Hz}$. Data acquisition and subsequent processing were performed using Acknowledge 3.5 software provided by BIOPAC Systems. Heart rate was derived from $R-R$ intervals and the power spectrum was calculated via the autocorrelation-FFT method to determine the LF band $(0.046 \mathrm{~Hz}-0.15 \mathrm{~Hz})$, $\mathrm{HF}$ band $(0.15 \mathrm{~Hz}-0.40 \mathrm{~Hz})$ and their ratio $(\mathrm{LE} / \mathrm{HF})$ as a parameter of sympatho-vagal balance.

An enzyme immunoassay kit (High Sensitity Salivary Cortisol Enzyme Immunoassay Kit ${ }^{\circledR}$, Salimetrics, U.S.A.) was used for the salivary cortisol assay process, according to the following procedure based on the manual. The system requires $50 \mu \mathrm{l}$ of saliva, along with a detector with a wavelength of $450 \mathrm{~nm}$ (Multiskan JX detector, Thermoelectron, U.S.A) for total cortisol

Ericsson's method (1959) was used to determine salivary buffering action. Hydrochloric acid was added to the saliva, and saliva $\mathrm{pH}$ was measured after the saliva was allowed to react for twenty minutes (Ericsson and Yngve, 1959). Salivary pH was measured using Duotest (Macherey-Nagel, Germany), with the test paper method ( $\mathrm{pH}$ ranges: 1.0-4.3, 3.5-6.8, 5.0-8.0, 7.2-10.0) (Kato et al., 2006).

Pleasantness and arousal were measured by using the Affect-grid method.

\section{Statistical analysis}

Statistical data are presented in the following form: " mean \pm standard deviation '. All $p$-values and confidence limits were based on two-tailed calculations. A $p$-value level of less than 0.05 was deemed significant. Analysis of variance (ANOVA) was used to analyze differences between the two postures, sitting and HDT, and anxiety status (high anxiety and normal). The ' $F$ statistic was used to indicate ratio differences between treatment and control (a parenthesis following $F$ shows ' factor, degree of freedom'). A simple main effect test based on least significant differences $(p=0.05)$ was used to examine the statistical significance of two-mean comparisons for paired samples. One participant in the high anxiety group for whom no saliva was collected with poor cortisol measurement accuracy were excluded from analysis. In addition, three participants who could not have precisely the LH/HF's measurement ( 1 in the high anxiety group, 2 in the normal group) were excluded from analysis.

\section{Results}

Concentration of salivary cortisol and Salivary buffering action

Figure 1 shows average salivary cortisol concentrations for the two groups in different postures. The high anxiety group had increased concentrations about 1.6 times under the HDT condition that were compared it with on the sitting posture, $F(1,25)=6.65$, $p<0.05$. In the other, the normal group did not show the remarkable increase by the posture conversion. In addition, the tendency significant difference was seen between two groups under the HDT condition, $F(1$, $25)=3.12, p<0.10$. Moreover, in the four factors tested using ANOVA, the interaction between high anxiety levels and the HDT posture was the most significance, $F(1$, $25)=5.25, p<0.05$. In addition, the tendency significant difference was seen between two groups under the HDT condition, $F(1,25)=3.12, p<0.10$.

No statistically significant differences in salivary buffering action were observed between the two groups or postures (Table 1.).

\section{Autonomic nervous system response}

Figure 2 shows the heart rate variability index (LF/HF ratios) ratios of the two groups in the different postures. High anxiety group showed increase about 5 times the LF /HF ratios under the HDT condition that were compared it with on the sitting posture, $F(1,23)=4.83, p<0.05$. Therefore the high anxiety group showed higher LF/HF

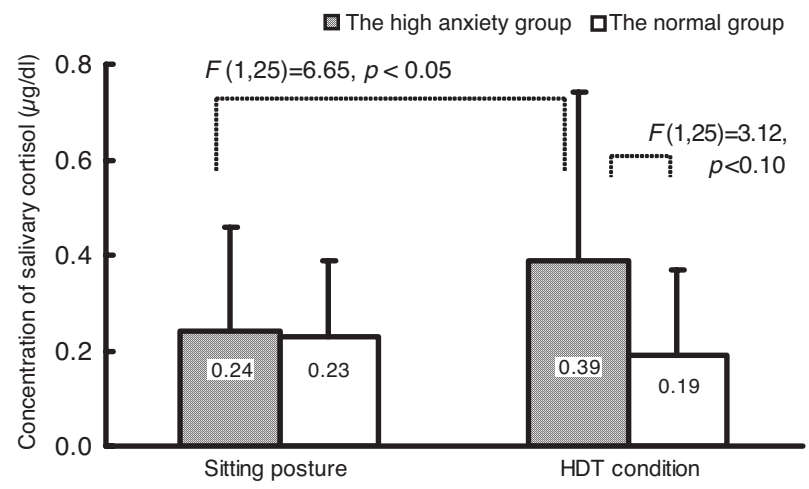

Fig. 1. Posture dependent cortisol concentrations.

The high anxiety group showed $0.24 \pm 0.22 \mu \mathrm{g} / \mathrm{dl}$ under sitting posture, and showed $0.39 \pm 0.35 \mu \mathrm{g} / \mathrm{dl}$ under HDT condition. 
Kato, M. et al.

Table 1 Results of Salivary buffering action, Arousalness score and Heart rate.

\begin{tabular}{|c|c|c|c|c|c|c|c|}
\hline \multirow[t]{2}{*}{ group } & \multirow[t]{2}{*}{ posture } & \multicolumn{2}{|c|}{$\begin{array}{l}\text { Salivary buffering } \\
\text { actions }\end{array}$} & \multicolumn{2}{|c|}{ Arousalness } & \multicolumn{2}{|c|}{ Haret rate } \\
\hline & & Mean & $S D$ & Mean & $S D$ & Mean & $S D$ \\
\hline \multirow{2}{*}{ The high anxiety group } & Sitting posture & 5.36 & 0.29 & 4.40 & 1.54 & 62.7 & 7.7 \\
\hline & HDT condition & 5.26 & 0.37 & 4.93 & 1.39 & 66.5 & 6.2 \\
\hline \multirow{2}{*}{ The normal group } & Sitting posture & 5.27 & 0.46 & 4.92 & 1.54 & 61.8 & 9.2 \\
\hline & HDT condition & 5.38 & 0.43 & 6.00 & 2.00 & 63.9 & 6.4 \\
\hline
\end{tabular}

ratios than in the normal group under the HDT condition, $F(1,23)=8.65, p<0.01$. In other, the normal group did not show the remarkable increase by the posture conversion. An interaction between high anxiety levels and posture was significant, $F(1,23)=4.46, p<0.05$. There was also a significant main effect of anxiety level, $F(1,23)=4.50$, $p<0.05$. The additional result showed significantly higher heart rate under the HDT condition than sitting position(Table 1).

\section{Affect-grid scores}

Figure 3 shows averaged affect-grid scores for the two groups. The high anxiety group held about 1.5 points of low pleasantness from time of the sitting posture after having moved to HDT condition $\{F(1,26)=5.66, p<0.05\}$. Furthermore, pleasantness ratings provided by the high anxiety group were significantly lower than those provided by the normal group in the HDT condition, $F(1,26)=15.99$, $p<0.01$.

Subjective arousal was consistently higher during the HDT condition than during the sitting posture, $F(1$, $26)=4.32, p<0.05$. In other words, the increase of the arousal score under the HDT condition was accepted with both the higher anxiety group and the normal group (Table 1).

\section{Discussion}

The present study evaluated psycho-physiological effects (autonomic responses indexed via heart rate variability and salivary cortisol) of two postures on

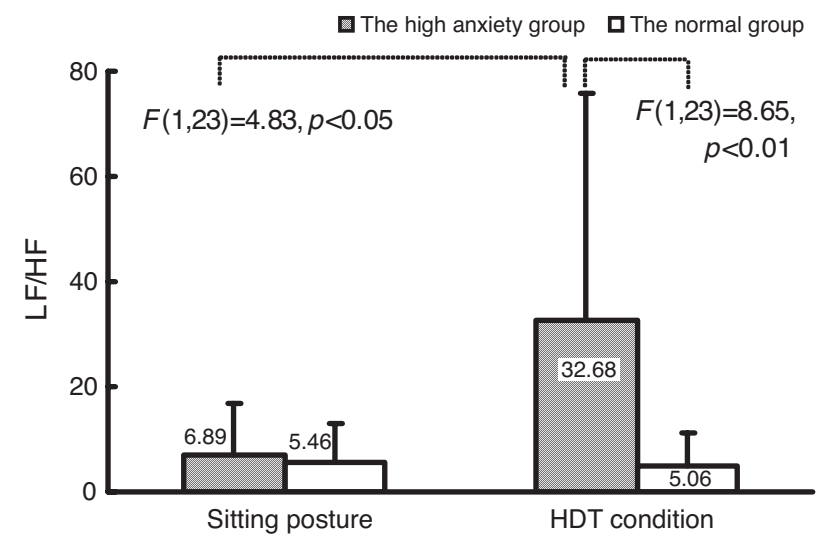

Fig. 2. Heart rate variability index (LF/ HF) calculated from heart rate power spectra for the two groups. emotional state. The high anxiety group showed a higher cortisol concentration under the HDT condition as compared to a sitting posture, and also showed higher LF/HF, a sympatho-vagal ratio indicating suppression of parasympathetic activity. Moreover, the high anxiety group provided significantly higher unpleasantness ratings compared to the normal group. It is noteworthy that all participants were mentally healthy students. The HDT appears to evoke a small and likely imperceptible change in gravitational environment that nevertheless exerts physiological and subjective stress on anxious individuals. In this study, the participants performed an attitude of a microgravity environment for only 20 minutes. In spite of such short time, the highly anxiety persons were affected by HDT. Earlier work suggests that highly anxiety persons easily experience stress, which can even influence such daily experiences as tasting food (Kato et al., 2006). The STAI may prove to be a useful component of a psychophysiological evaluation of gravitational tolerance and/or adaptability to a microgravity environment.

\section{Acknowledgements}

We thank Ms. Itoh for her support during data collection. Part of this work was supported by Daikouzaidan's Art and Science encouragement program.

\section{References}

Candland, D.K. (1962) Emotion. Bodily Change (ed. Candland, D.K.), London, D. Van Nostrand Co. Inc.

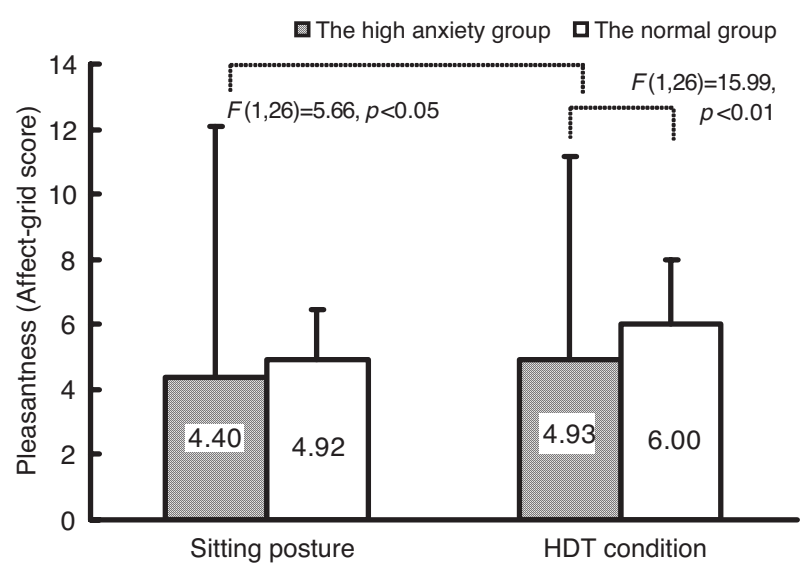

Fig. 3. Averaged affect-grid scores for pleasantness. 
Ericsson, and Yngve, (1959) Clinical Investigations of The Salivary Buffer Action, Acta Odontol. Scand., 17, 131165.

Iwase, S. (2002) Sympathetic adjustment to Microgravity exposure during space flight, Saishin igaku, 57, 10871098. [Article in Japanese]

Kamimura, E. (2001) Dictionary of Psychology (Nakajima,Y., Ando, K., Koyasu, M., Banno, Y., Shigemasu, K., Tathibana, M and Hakoda, Y., .ed), The yuhikaku Co., Tokyo. pp.739. [Article in Japanese]

Kato, M., Naga, S., and Shimizu, J. (2006) Salivary buffering action as an indicator of chronic stress, $J$. Anal. Bio-Sci., 29, 157-161.

Kato, M., Itoh, Y., Naga, S., and Shimizu, J., (2006) Effect of A-Trait personality on sweetness taste preferences, J. Integr. stud. diet. habits, 17, 44-48.[Article in Japanese]

Kawamura, Y. (1986) Physiology of oral cavity for dental students (de. Kawamura, Y.). Kyoto, Nagasueshoten. [Article in Japanese]

Mano, T. (2004) The orthostatic hypotension after spaceflight, Auton. nerv. syst., 41, 45-50.

Nakazato, K. (1982) The Japanese State-Trait anxiety inventory, Jpn.J.Psychosom. Med., 22, 107-112.

Shimizu, J., Naga, S., and Tamaru, M. (1999) Changes of cortisol level in saliva evoked by affective video film stimuli, Jpn. J. physiol. Psychol. Psychophysiol., 17, 74. [Article in Japanese]

Shimizu, T. (2000) The Microgravitational Environment and Blood Circulation, Resp. circ., 48, 453-461.

Spielberger, C.D. (1972) Anxiety as an emotional state. Anxiety - Current trends and theory(ed. Spielberger, C.D.). New York, Academic Press.

Russell, J.R., Weiss, A., and Mendelsohn, G.A. (1989) Affect-Grid: A Single-Item Scale of Pleasure and Arousal, J. Pers. Soc. Psychol., 57, 493-502. 